\title{
Interruption or Discontinuation of Tyrosine Kinase Inhibitor Treatment in Chronic Myeloid Leukaemia: A Retrospective Cohort Study (SPARKLE) in Belgium
}

\author{
Timothy Devos $^{\mathrm{a}} \quad$ Gregor Verhoef $^{\mathrm{a}} \quad$ Eva Steel $^{\mathrm{b}} \quad$ Dominiek Mazure $^{\mathrm{b}}$ \\ Philippe Lewalle ${ }^{c}$ Dominique Bron ${ }^{c}$ Zwi Berneman ${ }^{d}$ Fleur Samantha Benghiat ${ }^{\mathrm{e}}$ \\ Philippe Mineur $^{f}$ Koen Theunissen ${ }^{9}$ Pierre Zachée $^{h} \quad$ Chantal Doyen $^{i}$ Natalie Put ${ }^{j}$ \\ Marie Lejeune $^{k}$ Koen Van Eygen' Violaine Havelange ${ }^{m} \quad$ Michael Reusens $^{n}$ \\ Wim Pluymers $^{\mathrm{n}}$ Karen Peeters $^{\mathrm{n}}$

\begin{abstract}
${ }^{a}$ Hematology Department, University Hospitals Leuven, Leuven, Belgium; 'bniversitair Ziekenhuis Ghent, Ghent, Belgium; ' Institut Jules Bordet, Brussels, Belgium; ${ }^{\mathrm{d}}$ Universitair Ziekenhuis Antwerpen, Edegem, Belgium; ${ }^{\mathrm{e}} \mathrm{Hôp}$ ital Erasme, Brussels, Belgium; ' Grand Hôpital de Charleroi (GHdC), Charleroi, Belgium; ${ }^{9}$ Jessa Ziekenhuis, Campus

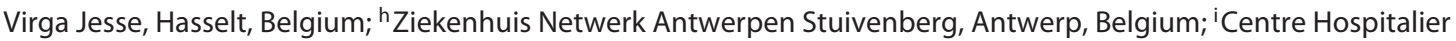
Universitaire UCL Namur, Yvoir, Belgium; 'Ziekenhuis Oost-Limburg, Campus Sint-Jan, Genk, Belgium; ${ }^{k}$ Centre Hospitalier Universitaire de Liège, Liège, Belgium; 'AZ Groeninge, Campus Kennedylaan, Kortrijk, Belgium; mUCL Saint-Luc,

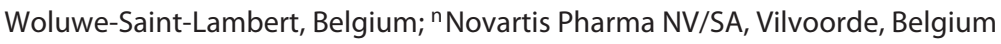

\section{Keywords}

Chronic myeloid leukaemia · Tyrosine kinase inhibitor .

Treatment interruption/discontinuation · Molecular

response · Imatinib · Nilotinib · Dasatinib · Ponatinib

\begin{abstract}
Objectives: To assess interruptions/discontinuations of tyrosine kinase inhibitor (TKI) treatment in Belgian patients with chronic myeloid leukaemia (CML). Methods: This retrospective study included patients with TKI interruptions/discontinuations of $\geq 4$ continuous weeks (no clinical trial context) between May 2013 and May 2016. Data collection took place between October 2016 and February 2017. Results: All 60 participants (69 interruptions/discontinuations) had chronic-phase CML and $75 \%$ had at least a major molecular response ( $\geq$ MMR) at interruption/discontinuation. Most interruptions/discontinuations occurred while on imatinib
\end{abstract}

(36/69; 49\%) and dasatinib (20/69; 29\%). Most interruptions/ discontinuations occurred due to side effects/intolerance (46/69; 67\%); other reasons included a wish to conceive $(6 / 69 ; 9 \%)$ and attempts to achieve treatment-free remission (TFR) $(6 / 69 ; 9 \%)$. Interruptions due to side effects occurred later for imatinib- or dasatinib-treated patients than for those on nilotinib or ponatinib. Treatment was re-initiated in $62 \%(43 / 69)$ of cases. Most interruptions caused by side effects/intolerance were followed by treatment changes. All 4 patients with $\geq$ MR 4.5 at interruption/discontinuation and $\geq 11$-month follow-up who had not restarted treatment maintained the response. Conclusion: Although TKIs are used for long-term CML treatment, physicians sometimes recommend interruptions/discontinuations. In this study, interruptions/discontinuations were mainly caused by side effects or intolerance, rather than TFR attempts.

\section{KARGER}

(C) 2019 S. Karger AG, Basel 


\section{Introduction}

Adenosine triphosphate-competitive tyrosine kinase inhibitors (TKIs) targeting BCR-ABL1, the driving oncoprotein of chronic myeloid leukemia (CML), revolutionized patient outcomes. After the introduction of imatinib in the early 2000s, the more potent second-generation drugs, dasatinib, nilotinib, and bosutinib, followed by the third-generation compound, ponatinib, enriched the therapeutic arsenal [1].

Treatment discontinuation in CML is relatively frequent and although there has been an increased focus on treatment-free remission (TFR) as a new goal of therapy in CML, the interruption or discontinuation of therapy can occur for a myriad of other reasons in the real-life setting, e.g., to avoid worrying side effects including those predisposing to other chronic disease, eliminate lowgrade chronic toxicity that substantially impacts a patient's quality of life, and be freed up from the daily constraints of medication intake. Other reasons could be a pregnancy or the wish to conceive, no longer needing to be careful about drug-drug/food-drug interactions, and the financial burden of targeted therapy [2-5].

It also needs to be noted that this broad variety of reasons to interrupt TKI treatment in CML are either recommended by the treating physician (e.g., in the case of a TFR attempt, severe side effects, toxicity, pregnancy, and the wish to conceive) or not (i.e., non-adherence, which can be related to side effects and other reasons). Optimal adherence to CML therapy is of key importance to maximize treatment effectiveness. Two clinical studies (ADAGIO and Hammersmith) have proven a clear correlation between adherence and achieving optimal treatment response, and they revealed that non-adherence is indeed common in CML patients $[6,7]$. In fact, it was demonstrated that non-adherence is associated with disease progression and a 3-foldhigher risk of poor treatment outcomes [7].

Although most CML patients need life-long TKI treatment, the promising results of imatinib interruption in a small number of cases [8-10] prompted the launching of discontinuation trials. Subsequently, in the multi-centre Stopping Imatinib (STIM) study [11-13], the CML8 (TWISTER) study from the Australasian Leukaemia and Lymphoma Group [14, 15], and the multi-centre observational According to Stop Imatinib (A-STIM) study [16], at least one-third of patients achieved TFR after interrupting treatment with imatinib. TFR clinical trials have also been conducted with second-generation TKIs, including ENESTfreedom [17], ENESTop [18], STOP 2G-TKI [19], DADI [20], and DASFREE [21]. Since mid-
2017, TFR is included in the label of nilotinib, based on the ENESTfreedom and ENESTop trials $[17,18]$. The use of second-generation TKIs does not seem to improve the results when compared to imatinib but does appear to impact on the median time from treatment start to the attempt to stop [22]. All the studies published so far clearly demonstrate that discontinuation is feasible and safe if performed in patients with a long-lasting deep molecular response (DMR), with no reports of disease progression or clonal evolution [22]. The last version of the $2013 \mathrm{Eu}-$ ropean LeukemiaNet recommendations suggested continuing TKI therapy indefinitely and that discontinuation would be appropriate only in a controlled clinical trial [23]. Hughes and Ross [24] reported for the first time in 2016 how to move TFR into the clinical practice. A position on TKI discontinuation was also taken by the NCCN in the new 2017 version of the CML guidelines [25].

In Belgium, national guidelines currently recommend staging of CML according to European LeukemiaNet [5, 25] or World Health Organisation [26] criteria, and initiating first-line treatment with imatinib, nilotinib, or dasatinib in chronic-phase (CP) patients, with careful monitoring of any adverse events (AEs) and potential drug interactions. Nilotinib, dasatinib, bosutinib, and ponatinib are considered for patients resistant or intolerant to their first-line TKI and also for advanced-phase patients. According to national guidelines, interruption or discontinuation of TKI treatment is recommended when severe skin rash or grade 3 hepatic toxicity occurs, or for female $\mathrm{CP}$ patients that have achieved a major molecular response (MMR) for $\geq 2$ years and are planning to conceive. TFR attempts are only recommended in clinical trial settings for patients in a deep sustained response [27].

Treatment discontinuation in CML is relatively frequent and, although there has been an increased focus on treatment free remission (TFR) as new goal of therapy in $\mathrm{CML}$, therapy interruption or discontinuation can be for a myriad of other reasons in the real-life setting. In the existing literature, only a limited amount of data is currently available on this matter. We therefore conducted this trial to characterise TKI interruptions or discontinuations in Belgian CML patients and examine the reasons for TKI interruption or discontinuation in real-life situations with related national guidelines.

\section{Methods}

Study Design and Participants

The study on the interruption or discontinuation of tyrosine kinase inhibitor treatment in chronic myeloid leukaemia, SPAR- 
KLE (A Belgian Retrospective Study on the Interruption or Discontinuation of Tyrosine Kinase Inhibitor Treatment in Chronic Myeloid Leukaemia) was a phase-IV, retrospective, observational, cross-sectional study based on data collected from chart reviews of CML patients from 13 Belgian haematology centres between October 2016 and February 2017. No treatment was administered as part of this study. All clinical decisions were made as per the treating physician's best clinical judgement.

Eligible participants were CML patients who had $\geq 1$ TKI interruptions/discontinuations (i.e., either interrupting temporarily or with the intention to discontinue indefinitely) of $\geq 4$ continuous weeks (regardless of the reason) outside the context of a clinical trial between 31 May 2013 and 31 May 2016. Patients with an interruption of $<4$ weeks were excluded to avoid evaluating those whose treatment was interrupted only briefly because of transient toxicity. We only included patients with a TKI interruption/discontinuation of $\geq 4$ continuous weeks. As such, the study detected only the persistent phase of adherence according to the taxonomy of Vrijens et al. [28] (i.e., when a prescribed therapy is discontinued early), but not the non-implementation phase of adherence which may be more common in CML (i.e., the prescribed therapy is still taken but not in a fully compliant manner).

\section{Study Objectives}

The objectives of this observational study were to assess the predefined characteristics of patients and other parameters of TKI treatment interruption/discontinutation. A post hoc sub-group analysis was carried out, in patients followed for at least 11 months, of treatment interruptions/discontinuations that occurred between May 2013 and May 2015.

The predefined characteristics of patients and other outcomes of TKI treatment interruption/discontinuation were collected and analysed. The information collected as per protocol was inserted into an electronic case report form; no personally identifiable data was included.

Captured data consisted of the characteristics of patients, treatment interruption, and, if applicable, reinitiation of treatment. Patient characteristics included age, gender, Sokal risk score, CML phase, and disease duration. Characteristics of the treatment interruption included the duration and reason of the TKI interruption; the duration, type and line of TKI therapy that was interrupted; the time between TKI interruption and the first BCR-ABL1 RT-qPCR molecular test; and the depth of (molecular) response of the patient and its duration prior to TKI interruption.

Five levels of TKI response were used in this study: a complete haematological response (CHR), a complete cytogenetic response (CCyR), and 3 levels of MR, namely, MMR (also referred to as MR3; a 3-log reduction from the standardised baseline or $\leq 0.1 \%$ $B C R-A B L 1$ on the International Scale as assessed by quantitative reverse-transcriptase PCR), MR4 (a 4-log reduction from the standardised baseline or $\leq 0.01 \% B C R-A B L 1$ ), and MR4.5 (a 4.5-log reduction from the standardised baseline or $\leq 0.0032 \% B C R-A B L 1$ ) or deeper [20].

\section{Outcomes}

If the patient had reinitiated therapy, the outcomes of the treatment interruption/discontinuation that were captured included the date of reinitation and the depth of the response as well as the reasons for reinitation and the type of TKI used.

Interruption or Discontinuation of TKI Treatment in CML

Table 1. Whole-population analysis of $60 \mathrm{CML}$ patients who interrupted or discontinued TKI treatment

n

Whole population

In the chronic phase of CML

Female

Sokal risk score

Low

Intermediate

High

Not available

Mean age at latest interruption/discontinuation

$>61$ years $\quad 33$

$\%$

100

29

17

20

1

22

27

33

Response at the time of TKI treatment interruption/ discontinuation ${ }^{1}$

CHR

CCyR

MMR

MR4

MR4.5 or deeper

$\begin{array}{rr}15 & 22 \\ 2 & 3 \\ 16 & 23 \\ 11 & 16 \\ 25 & 36\end{array}$

${ }^{1}$ Nine patients each had 2 treatment interruptions/discontinuations.

\section{Statistical Methods}

All analyses were performed with macro-enriched Microsoft Excel 2016 MSO (16.0.8201.2102) 32-bit version. They were performed on the overall population (for most outcomes), the subset of patients who interrupted/discontinued treatment due to pregnancy or the wish to conceive, and the subset of patients followed for at least 11 months.

All analyses were descriptive, and no statistical hypotheses were formulated. Missing data was excluded from the database when calculating percentages of patients or interruptions.

Some patients had multiple TKI treatment interruptions/discontinuations, so the number of interruptions/discontinuations was higher than the number of participants. Most analyses used variables related to interruptions/discontinuations (69 cases), so the number of interruptions/discontinuations was considered for calculating the statistical parameters instead of the number of participants $(n=60)$.

A contract research organization, XPE Pharma \& Science, performed the statistical analysis under the supervision of the sponsor, Novartis.

\section{Results}

\section{Study Population}

Sixty CML patients participated in the study; 51 had 1 and 9 had 2 TKI interruptions/discontinuations, respectively (Table 1 ). The total number of cap- 
Table 2. Number of interruptions/discontinuations (I/Ds) according to type and line of treatment received prior to interruption/ discontinuation in 69 patients (whole-population analysis)

\begin{tabular}{lcc}
\hline & I/Ds, $n$ & I/Ds, \% \\
\hline Imatinib & & \\
As 1st-line & 23 & 33 \\
As line $\geq 2$ & 11 & 16 \\
Nilotinib & & \\
$\quad$ As 1st-line & 3 & 4 \\
As line $\geq 2$ & 9 & 13 \\
Dasatinib & & 3 \\
As 1st-line & 2 & 26 \\
As line $\geq 2$ & 18 & \\
Ponatinib & & 4 \\
As line $\geq 2$ & 3 &
\end{tabular}

tured TKI interruptions/discontinuations was therefore 69.

Patients were homogeneously distributed by gender; mean age was 61.0 years. All patients had CP CML. The Sokal risk score was low/intermediate in 61\% (37/60) of patients, high in $2 \%(1 / 60)$, and not available in $37 \%$ $(22 / 60)$.

Of all treatment interruptions/discontinuations, 36\% $(25 / 69)$ occurred in patients with MR4.5 or deeper, $16 \%$ (11/69) in patients with MR4, 23\% (16/69) in patients with MMR, and 25\% (17/69) in patients without at least an MMR.

\section{Treatment Interruptions/Discontinuations}

The median time between CML diagnosis and TKI treatment interruption/discontinuation was 363 weeks; patients were treated with TKIs (i.e., the specific TKI treatment that was interrupted/discontinued) for a median time of 169 weeks before the interruption/discontinuation.

Most TKI interruptions/discontinuations occurred while on imatinib or dasatinib, i.e., $49 \%(34 / 69)$ in patients taking imatinib and 29\% (20/69) in patients taking dasatinib (the latter occurred mostly when taken as second-line treatment) (Table 2).

The duration of the MR prior to TKI treatment interruption/discontinuation in the overall population ranged between 0 and 615 weeks; the median duration was 37 weeks. The respective durations of the MR prior to TKI interruption/discontinuation are described according to sub-characteristics in online supplementary Table 1 (for all online suppl. material, see www.karger. com/doi/10.1159/000499329).
The main reasons for TKI treatment interruption/discontinuation were side effects or intolerance (in 46/69 cases [67\%]), TFR attempts (6/69 [9\%]), and the wish to conceive $(6 / 69$ [9\%]). Of the interruptions/discontinuations due to side effects or intolerance, no significant influence of gender and age was observed (online suppl. Table 2).

For 17\% (12/69) and 25\% (17/69) of the interruptions/ discontinuations, patients had a low and intermediate Sokal risk score, respectively. For 1 interruption/discontinuation, the patient had a high Sokal risk score, and for $23 \%(16 / 69)$ no score was not available. The median duration of the treatment interruption/discontinuation was 112 days (Table 3 ).

Twenty-eight of the 69 (41\%) TKI interruptions/discontinuations were in patients on first-line TKI treatment, and a comparable number $(25 / 69 ; 36 \%)$ in patients on second-line therapy (online suppl. Table 2).

Interruptions/discontinuations of imatinib treatment were the longest (median duration 186 days) when compared to other TKIs. The median duration of treatment interruption/discontinuation was longest in patients wishing to conceive (253 days) and shortest if they took place at the request of the patient (59 days). Interruptions/discontinuations due to side effects or intolerance lasted for a median 98 days, although this varied greatly, from 29 to 904 days.

Interruptions/discontinuations lasted a median of 24 weeks in study participants with an MMR (approx. 21 and 17 weeks in participants with MR4 and MR4.5 or deeper, respectively), while in those with $\mathrm{CHR}$ and $\mathrm{CCyR}$, they lasted a median of 13 and 10 weeks, respectively (Table $3)$. The median follow-up time between interruption/discontinuation and first molecular test did not vary much with different reasons for interruption/discontinuation (56 days for those due to side effects or intolerance, 49 days for TFR attempts, 47 days in patients wishing to conceive, and 48 days upon patient request). Those with less than MMR at treatment interruption/discontinuation had a shorter median time between interruption/discontinuation and first molecular test (Table 4).

Of the 34 patients (40 interruptions/discontinuations) that were followed for at least 11 months after interrupting or discontinuing TKI treatment, 7 were still off treatment at the end of the study. Of these 7, all 4 with an MR4.5 or deeper response at the time of discontinuation maintained this response, 2 with MR4 at the time of discontinuation had an MMR, and 1 with MMR at the time of discontinuation had CCyR at the last follow-up. 
Table 3. Duration of TKI treatment interruption/discontinuation overall and per sub-characteristic, and reason for interruption/discontinuation in 69 patients (whole-population analysis)

\begin{tabular}{|c|c|c|c|c|}
\hline & \multirow[t]{2}{*}{$n$} & \multicolumn{3}{|c|}{ Duration, days } \\
\hline & & mean & median & range \\
\hline Chronic phase of CML & 69 & 175.7 & 112 & $29-904$ \\
\hline Female gender & 33 & 173.6 & 107 & $32-904$ \\
\hline \multicolumn{5}{|l|}{ Sokal risk score } \\
\hline Low & 18 & 237.8 & 141 & $33-904$ \\
\hline Intermediate & 25 & 140.6 & 89 & $32-833$ \\
\hline High & 1 & 345.0 & 345 & $345-345$ \\
\hline Not available & 25 & 159.4 & 140 & $29-536$ \\
\hline \multicolumn{5}{|l|}{ Age at latest interruption/discontinuation ${ }^{1}$} \\
\hline$\leq 64$ years $($ median $)$ & 33 & 184.2 & 132 & $29-833$ \\
\hline$>64$ years (median) & 36 & 168.0 & 94 & $32-904$ \\
\hline$\leq 61$ years $($ mean $)$ & 30 & 191.3 & 136 & $29-833$ \\
\hline$>61$ years $($ mean $)$ & 39 & 163.8 & 96 & $32-904$ \\
\hline \multicolumn{5}{|l|}{ Line of TKI treatment } \\
\hline 1st & 28 & 239.8 & 184 & $29-904$ \\
\hline 2 nd & 25 & 133.3 & 85 & $33-425$ \\
\hline $3 \mathrm{rd}$ & 4 & 126.3 & 113 & $91-189$ \\
\hline 4 th & 4 & 293.5 & 263 & $112-536$ \\
\hline 5 th & 5 & 54.8 & 43 & $35-105$ \\
\hline 6 th & 3 & 42.3 & 44 & $36-47$ \\
\hline \multicolumn{5}{|c|}{ TKI received prior to interruption/discontinuation } \\
\hline Imatinib & 34 & 214.9 & 186 & 29-904 \\
\hline Nilotinib & 12 & 168.7 & 89 & $36-833$ \\
\hline Dasatinib & 20 & 130.3 & 85 & $33-686$ \\
\hline Bosutinib & 0 & - & - & - \\
\hline Ponatinib & 3 & 63.7 & 44 & $35-112$ \\
\hline Other & 0 & - & - & - \\
\hline \multicolumn{5}{|c|}{ Interval between CML diagnosis and treatment interruption/discontinuation ${ }^{1}$} \\
\hline$\leq 363$ weeks (median) & 35 & 136.4 & 91 & $29-833$ \\
\hline$>363$ weeks (median) & 34 & 216.3 & 183 & $35-904$ \\
\hline$\leq 426$ weeks (mean) & 39 & 150.6 & 91 & $29-833$ \\
\hline$>426$ weeks (mean) & 30 & 208.5 & 183 & $35-904$ \\
\hline \multicolumn{5}{|c|}{ Duration of the TKI treatment that was interrupted/discontinued ${ }^{1}$} \\
\hline$\leq 169$ weeks (median) & 35 & 180.7 & 91 & $32-904$ \\
\hline >169 weeks (median) & 34 & 170.6 & 148 & $29-536$ \\
\hline$\leq 238$ weeks (mean) & 42 & 168.0 & 91 & $32-904$ \\
\hline$>238$ weeks (mean) & 27 & 187.7 & 185 & $29-536$ \\
\hline \multicolumn{5}{|c|}{ Response at the time of TKI treatment interruption/discontinuation } \\
\hline CHR & 15 & 107.3 & 91 & $32-345$ \\
\hline CCyR & 2 & 70.5 & 71 & $36-105$ \\
\hline MMR & 16 & 199.2 & 168 & $35-833$ \\
\hline MR4 & 11 & 233.9 & 149 & $33-686$ \\
\hline MR4.5 or deeper & 25 & 184.6 & 124 & 29-904 \\
\hline \multicolumn{5}{|c|}{ Reason for treatment interruption/discontinuation interruption/discontinuation } \\
\hline TFR attempt & 6 & 174.2 & 186 & $36-358$ \\
\hline A wish to conceive & 6 & 271.0 & 253 & $165-420$ \\
\hline Spontaneous request of the patient & 2 & 58.5 & 59 & $32-85$ \\
\hline Due to side effects or intolerance & 46 & 166.1 & 98 & $29-904$ \\
\hline Other & 9 & 188.7 & 96 & $42-833$ \\
\hline
\end{tabular}


Table 4. Median time between treatment interruption/discontinuation (I/D) and first molecular test

\begin{tabular}{lcc}
\hline & $I / D s, n$ & Median time, days \\
\hline Overall & 62 & 51 \\
\hline Depth of best response at the time of treatment interruption/discontinuation (or the most recent test) \\
$<$ MMR & 13 & 37 \\
MMR & 14 & 77 \\
MR4 & 11 & 52 \\
MR4.5 or deeper & 24 & 58 \\
\hline Reason for treatment interruption/discontinuation & & \\
TFR attempt & 6 & 49 \\
A wish to conceive & 6 & 47 \\
Spontaneous request of the patient & 2 & 48 \\
Due to side effects or intolerance & 41 & 56 \\
Other & 7 & 71 \\
\hline
\end{tabular}

Table 5. Exploratory statistics on various parameters according to TKI before treatment interruption/discontinuation for patients who interrupted/discontinued the treatment due to side effects (whole-population analysis)

\begin{tabular}{|c|c|c|c|c|c|c|c|c|}
\hline \multirow[t]{2}{*}{$\begin{array}{l}\text { TKI received } \\
\text { prior to I/D }\end{array}$} & \multicolumn{4}{|c|}{ Overall $^{\mathrm{a}}$} & \multicolumn{2}{|c|}{$\begin{array}{l}\text { Patients who } \\
\text { restarted treatment }^{\mathrm{b}}\end{array}$} & \multicolumn{2}{|c|}{$\begin{array}{l}\text { Patients who restarted } \\
\text { treatment with a different TKI }\end{array}$} \\
\hline & $n$ & $\%$ & $\begin{array}{l}\text { treatment duration } \\
\text { before } I / D \text {, weeks }{ }^{\mathrm{d}}\end{array}$ & $\begin{array}{l}\text { duration of } \\
\mathrm{I} / \mathrm{D} \text {, days } \mathrm{s}^{\mathrm{d}}\end{array}$ & $n$ & $\%$ & $n$ & $\%$ \\
\hline Imatinib & 20 & 43 & 268 & 165 & 13 & 38 & 5 & 28 \\
\hline Nilotinib & 6 & 13 & 9 & 65 & 6 & 18 & 4 & 22 \\
\hline Dasatinib & 17 & 37 & 51 & 84 & 13 & 38 & 8 & 44 \\
\hline Bosutinib & 0 & - & - & - & 0 & - & 0 & - \\
\hline Ponatinib & 3 & 7 & 4 & 44 & 2 & 6 & 1 & 6 \\
\hline Other & 0 & - & - & - & 0 & - & 0 & - \\
\hline
\end{tabular}

No treatment interruptions/discontinuations were recorded for bosutinib or other TKIs. I/D, interruption/ discontinuation; $n$, number of treatment interruptions/discontinuations (overall) or treatment reinitiations (restarted treatment).

${ }^{\mathrm{a}} n=46 ;{ }^{\mathrm{b}} n=34 ;{ }^{\mathrm{c}} n=18 ;{ }^{\mathrm{d}}$ median value.

Treatment Interruptions/Discontinuations Due to

Side Effects

In total, $67 \%(46 / 69)$ of interruptions/discontinuations were due to TKI side effects or intolerance (online suppl. Table 2). These occurred mostly while the patients were taking imatinib $(20 / 46 ; 43 \%)$ or dasatinib (17/46; $37 \%)$ (Table 5). Among patients reinitiating treatment after interrupting/discontinuing due to side effects, more reinitiations with TKI switching occurred for dasatinib $(8 / 17 ; 47 \%)$ than for imatinib $(5 / 20 ; 25 \%)$. Treatment interruptions/discontinuations due to side effects seemed to occur later in patients on imatinib (median 268 weeks) than in those on dasatinib (51 weeks), but then lasted longer (165 vs. 84 days). Very early treatment interruptions/ discontinuations (after a median of 9 and 4 weeks, respectively) due to side effects were observed for nilotinib and ponatinib.

Treatment Interruptions/Discontinuations Due to Pregnancy or the Wish to Conceive

In total, 6 patients interrupted/discontinued treatment due to their wish to conceive, 3 men and 3 women (Table 6). All were younger than 40 years, in the CP of CML. Three had a low Sokal risk score and 1 an intermediate Sokal risk score; no data was available for the remaining 2. All patients interrupting/discontinuing treatment to conceive had at least MMR at the time of interruption/discontinuation: 4/6 with an MMR, 1 with an 
Table 6. Summary of results for 6 patients who interrupted/discontinued treatment due to a pregnancy or the wish to conceive

\begin{tabular}{|c|c|c|}
\hline Characteristics & $n$ & $\%$ \\
\hline Chronic phase of CML & 6 & 100 \\
\hline Female gender & 3 & 50 \\
\hline \multicolumn{3}{|l|}{ Sokal risk score } \\
\hline Low & 3 & 50 \\
\hline Intermediate & 1 & 17 \\
\hline Not available & 2 & 33 \\
\hline \multicolumn{3}{|l|}{ Age at interruption/discontinuation } \\
\hline$\leq 40$ years & 6 & 100 \\
\hline \multicolumn{3}{|l|}{ Line of TKI treatment } \\
\hline 1 st & 3 & 50 \\
\hline 2nd & 2 & 33 \\
\hline $3 \mathrm{rd}$ & 1 & 17 \\
\hline \multicolumn{3}{|c|}{ TKI received prior to interruption/discontinuation ${ }^{\mathrm{a}}$} \\
\hline Imatinib & 4 & 67 \\
\hline Nilotinib & 2 & 33 \\
\hline \multicolumn{3}{|c|}{$\begin{array}{l}\text { Depth of response at the time of TKI treatment } \\
\text { interruption/discontinuation }\end{array}$} \\
\hline MR3 (MMR) & 4 & 67 \\
\hline MR4 & 1 & 17 \\
\hline MR4.5 or deeper & 1 & 17 \\
\hline \multicolumn{3}{|l|}{ Treatment reinitiated } \\
\hline No & 4 & 67 \\
\hline Yes (due to a loss of response) & 2 & 33 \\
\hline \multicolumn{3}{|c|}{ Treatment reinitiated with the same TKI } \\
\hline Yes & 2 & 33 \\
\hline \multicolumn{3}{|c|}{ Depth of response at the time of TKI treatment reinitiation ${ }^{\mathrm{b}}$} \\
\hline $\mathrm{CHR}$ & 1 & 50 \\
\hline No $\mathrm{CHR}^{\mathrm{c}}$ & 1 & 50 \\
\hline \multicolumn{3}{|c|}{$\begin{array}{l}\text { a All patients were receiving either imatinib or nilotinib. } \\
\text { b In a total of } 2 \text { patients. } \\
{ }^{c} \text { Complete relapse/no response/a loss of CHR. }\end{array}$} \\
\hline
\end{tabular}

MR4, and 1 with an MR4.5 or deeper. At the time of treatment interruption/discontinuation, 4/6 patients were using imatinib. Only 2 subsequently reinitiated treatment, both due to a loss of response. One had a complete relapse (no CHR) and the other had CHR before reinitiation. Both reinitiated treatment with the TKI used before interruption (i.e., 1 with imatinib and 1 with nilotinib).

The median duration of interruption/discontinuation of TKI treatment among these patients was 253 days. All patients wishing to conceive interrupted/discontinued the treatment for $\geq 5$ months.

Interruption or Discontinuation of TKI Treatment in CML

\section{Treatment ReInitiations}

Treatment was reinitiated after $62 \%$ (43/69) of interruptions/discontinuations, 20 of which were reinitiated with a different TKI from the one used before interruption/discontinuation (Table 7). Most reinitiations (34/43; $79 \%$ ) occurred following a treatment interruption/discontinuation due to side effects or intolerance. Of the patients on imatinib before the interruption who reinitiated treatment, 18\% (6/34) switched to a different TKI. Patients previously on nilotinib, dasatinib, and ponatinib reinitiated with a different TKI in $42 \%(5 / 12), 40 \%(8 / 20)$, and $33 \%(1 / 3)$ of cases, respectively. Among patients who reinitiated treatment with a different TKI, most interruptions/discontinuations were due to side effects or intolerance to the previous TKI.

None of the patients who interrupted TKI treatment for a TFR attempt had their treatment reinitiated $(0 / 6)$. Of the 6 who interrupted the treatment due to the wish to conceive, $33 \%(2 / 6)$ had treatment reinitiated. One of 2 patients who had interrupted TKI treatment by spontaneous request had treatment reinitiated. Of the patients that had interrupted TKI treatment due to side effects or intolerance, $74 \%$ (34/46) had treatment reinitiated.

The reasons for treatment reinitiation were either loss of response in $47 \%(20 / 43)$ of the cases, the resolution of side effects in $37 \%(16 / 43)$, and another reason in $16 \%$ (7/43).

Of the 40 interruptions/discontinuations that were followed up for at least 11 months, 33 treatment reinitiations were reported until study end. These included $4 \mathrm{pa}-$ tients with an MR4.5 or deeper at the time of discontinuation who reinitiated treatment due to a resolution of side effects but maintained their response.

Patients who reinitiated treatment had higher $B C R$ $A B L 1$ expression at the time of reinitiation than at the time of treatment interruption/discontinuation (not shown).

\section{Discussion}

This is the first study to assess interruptions/discontinuations of TKI treatment in Belgian CML patients in a real-life setting. All 60 patients with TKI interruptions/ discontinuations were in the CP of CML, mainly with a low or intermediate Sokal risk score. Out of a total of 69 TKI interruptions/discontinuations recorded, more than three-quarters occurred while on first- or second-line treatment, and nearly half while on imatinib. These findings are consistent with the fact that imatinib is a well-

Acta Haematol 2019;142:197-207 
Table 7. Exploratory statistics on various parameters according to TKI before treatment interruption/discontinuation (whole population analysis)

\begin{tabular}{|c|c|c|c|c|c|c|c|c|}
\hline \multirow[t]{2}{*}{$\begin{array}{l}\text { TKI received } \\
\text { prior to } \mathrm{I} / \mathrm{D}\end{array}$} & \multirow[t]{2}{*}{ Reason for I/D } & \multicolumn{3}{|c|}{ Overall population $^{\mathrm{a}}$} & \multicolumn{2}{|c|}{$\begin{array}{l}\text { Patients who } \\
\text { restarted treatment }{ }^{\mathrm{b}}\end{array}$} & \multicolumn{2}{|c|}{$\begin{array}{l}\text { Patients who restarted } \\
\text { treatment with a different } \mathrm{TKI}^{\mathrm{c}}\end{array}$} \\
\hline & & $n$ & $\%$ & $\begin{array}{l}\text { median I/D } \\
\text { duration, days }\end{array}$ & $n$ & $\%$ & $n$ & $\%$ \\
\hline \multirow[t]{3}{*}{ Imatinib } & TFR attempt & 5 & 7 & 189 & 0 & 0 & 0 & - \\
\hline & A wish to conceive & 4 & 6 & 282 & 1 & 2 & 0 & - \\
\hline & Other & 4 & 6 & 161 & 3 & 7 & 1 & 5 \\
\hline \multirow[t]{4}{*}{ Nilotinib } & TFR attempt & 1 & 1 & 182 & 0 & - & 0 & - \\
\hline & A wish to conceive & 2 & 3 & 227 & 1 & 2 & 0 & - \\
\hline & Spontaneous request of the patient & 1 & 1 & 85 & 1 & 2 & 0 & - \\
\hline & Due to side effects or intolerance & 6 & 9 & 65 & 6 & 14 & 4 & 20 \\
\hline Ponatinib & Due to side effects or intolerance & 3 & 4 & 44 & 2 & 5 & 1 & 5 \\
\hline
\end{tabular}

No I/Ds were recorded for bosutinib or other TKIs. I/D, interruption/discontinuation; $n$, number of treatment I/Ds (overall) or reinitiations (restarted treatment).

${ }^{\mathrm{a}} n=69 ;{ }^{\mathrm{b}} n=43 ;{ }^{\mathrm{c}} n=20$.

established treatment option in the front-line CML setting in Belgium.

Although TKI treatment is currently viewed as a lifelong treatment, discontinuing or interrupting it is considered in certain cases, e.g., when side effects impact severely on the quality of life, or the case of a pregnancy or the wish to conceive. Health care costs can also be a limiting factor. So far, cessation of treatment is not yet daily clinical practice for physicians. Current recommendations state that following TKI treatment stop, the MR should be monitored once per month during the first 6-12 months, although only 4 analyses per year are reimbursed by the Belgian health authorities [5, 24]. An MMR is considered optimal for survival, but to achieve a successful discontinuation of treatment, a sustained DMR is required [25]. In our survey, the MR at the time of treatment discontinuation/interruption was MMR or deeper in three-quarters of the cases. These MRs at the time of treatment discontinuation/interruption were achieved after a median of 85,27 , and 63 weeks, respectively, in patients with MMR, MR4, and MR4.5.

The main reason for TKI treatment interruption/discontinuation identified in our study was the presence of side effects or intolerance; however, treatment was later reinitiated in three-quarters of these cases, and with a dif- ferent TKI in 39\%. Most interruptions/discontinuations due to side effects or intolerance occurred in patients on imatinib (43\%) and dasatinib (37\%). This reflects current practice because most patients were being treated with imatinib or dasatinib at the time of the study. Less than one-tenth of the interruptions/discontinuations occurred due to a TFR attempt; none of these patients reinitiated treatment for the duration of our study.

The median duration of TKI treatment interruption/ discontinuation depended on the response at the time of interruption/discontinuation, with the low responders (CHR and CCyR) having a shorter interruption/discontinuation and a quicker monitoring of the response after treatment interruption/discontinuation than participants with at least MMR. The TKI treatment interruption/discontinuation was longer for patients with at least MMR, diagnosed for a longer time, and treated for $>1$ year with a first- or second-line TKI. The longest interruptions/discontinuations were recorded for patients on imatinib, which is also in line with the fact that imatinib was the most widely used TKI at the time of this study.

Following almost two-thirds of TKI interruptions/discontinuations, treatment was reinitiated. As expected, if treatment was interrupted/discontinued due to side effects or intolerance, it was then reinitiated (in almost 
three-quarters of cases). Three-quarters of these reinitiations occurred with a different TKI. Most low responders and patients with an MMR reinitiated treatment. The treatment reinitiation rate was higher in patients with a shorter interval between CML diagnosis and TKI treatment interruption/discontinuation and a shorter duration of TKI treatment. This is consistent with the current knowledge of successful TFR.

At treatment reinitiation, no CHR was reported in $19 \%$ of the cases; a CHR or CCyR was reported in about half of the cases.

The main reason for TKI treatment reinitiation was the loss of response after treatment interruption/discontinuation or the resolution of side effects. The first was more frequent in patients with MMR or a better response at the time of the interruption/discontinuation, while the latter occurred more frequently in patients with a low response. Of note, no CML patients developed acceleratedphase CML or a blast crisis; all remained in CP-CML.

As expected, follow-up of MR after interruption/discontinuation was fast (an overall median time of 51 days). However, this is slightly longer than the 1-month interval currently recommended in Belgian guidelines in cases of TFR attempts and can probably be partly attributed to the lack of reimbursement.

Both male and female participants interrupted/discontinued treatment in an attempt to conceive. Although the impact of TKI treatment on fertility or pregnancy outcome is not fully elucidated, there is no formal contraindication for procreation while on imatinib and dasatinib in male patients $[29,30]$. However, female patients should discontinue TKI treatment during conception and pregnancy, and all pregnancies should be planned to achieve at least an MMR before interruption/discontinuation. In our study, all patients wishing to conceive had an MMR or better response, and only one-third reinitiated treatment during the time the survey was conducted, due to a loss of response. Currently, Belgian experts recommend a treatment interruption/discontinuation of $\geq 3$ months to attempt procreation [27]; in our study, the median length of interruption/ discontinuation was 253 days (approx. 8.3 months).

Even though TKI therapies in CML are considered a life-long treatment, this study has shown that in a real-life setting, interruption or discontinuation occurs quite frequently in the CML population and for a variety of reasons.

The proportion of patients who interrupted treatment to attempt a long-term TFR was very small in this study ( $9 \%$ of all cases). This is in line with the fact that national guidelines as well as the most recent ELN guidelines of

Interruption or Discontinuation of TKI

Treatment in CML
2013, applicable at the time of TKI interruption of the patients in this study, suggest continuing TKI therapy indefinitely and that discontinuation is appropriate only in a controlled clinical trial [5].

A few patients interrupted TKI treatment due to the wish to conceive ( $9 \%$ of all cases). Indeed, conception and pregnancy are contraindicated during TKI treatment [5]. Belgian national guidelines also state that female patients who wish to conceive are advised to discontinue any TKI during conception and pregnancy [27].

The presence of side effects or intolerance was the most frequently reported reason to interrupt or discontinue TKI treatment in our study (67\% of all cases). The management of side effects is increasingly important as therapy is potentially for life and multiple TKIs are available [2]. Belgian national guidelines recommend discontinuing TKI therapy in cases of severe hepatic toxicity, severe skin rash, pancreatitis, and pulmonary hypertension [27]. In addition, it is known that low-grade chronic toxicities that substantially impact a patient's quality of life (and for other reasons) can lead to non-adherence which is commonly reported in CML. The 2013 ELN recommendations state that a reduction or interruption of treatment must only be done if optimal management of the AE cannot be accomplished in other ways, and frequent monitoring is needed to detect resolution of the $\mathrm{AE}$ as early as possible [23].

Eight patients with a follow-up of at least 11 months had MR4.5 or deeper at discontinuation; 4 of them restarted treatment due to a resolution of side effects while maintaining their response and 4 remained with MR4.5 and did not restart TKI treatment at the end of the study.

This retrospective study had several limitations. First, to set a threshold for significant treatment interruptions/ discontinuations in terms of duration, patients needed to have a TKI interruption/discontinuation of at least 4 continuous weeks to be included in the study. As such, we were not able to collect any real-life data on shorter TKI interruptions/discontinuations, which are also frequently observed in CML and contribute to non-adherence. We also did not make a distinction between therapy interruptions that were recommended and those not recommended by the treating physician. The study was retrospective, with a limited follow-up time (i.e., the interval between the TKI interruption and data collection). This meant that some variables were not captured for patients who were still off therapy at time of data collection. The kinetics of the response after the TKI interruption in the function of time were also not captured. No information was collected about the TKI dose being taken by patients at

Acta Haematol 2019;142:197-207 205 
the time of therapy interruption. This Belgian study included only 60 patients and provided descriptive data only and should be interpreted as such. Nonetheless, it study contains interesting real-life practice data complementing that collected in clinical trials of CML.

In conclusion, the main reasons for TKI interruption/ discontinuation in this retrospective study were side effects of or intolerance to the TKI, with TFR attempts representing only a small percentage. Irrespective of the reason for TKI interruption, almost half of the patients reinitiated treatment due to a loss of response. The results of this study underline the need of an individual approach for the optimal management of CML patients.

\section{Acknowledgements}

Medical writing and editorial support were provided by Lidia Malinas and Kristel Vercauteren (XPE Pharma \& Science c/o Novartis Pharma NV/SA). The authors would like to thank Lidia Malinas (XPE Pharma \& Science c/o Novartis Pharma NV/SA) for statistical support.

\section{Statement of Ethics}

In Belgium, retrospective studies fall outside clinical trial legislation and therefore do not require approval by a medical ethics committee. However, a notification was submitted to Independent Ethics Committees at each centre and they all provided their agreement or approval. Patients signed an informed consent sheet.

\section{Disclosure Statement}

M.R., W.P., and K.P. are employed by Novartis. T.D. reports consultancies for Ablynx, Gilead, and Novartis. G.V., E.S., D.M., P.L., D.B., Z.B., F.S.B., P.M., K.T., P.Z., C.D., N.P., M.L., and V.H. report no conflicts of interest. K.K.V.E. reports consultancy for Janssen and grants outside the submitted work for Roche and Janssen.

\section{Funding Source}

This work was supported by Novartis Pharma NV/SA, Belgium.

\section{References}

1 Chopade P, Akard LP. Improving Outcomes in Chronic Myeloid Leukemia over Time in the Era of Tyrosine Kinase Inhibitors. Clin Lymphoma Myeloma Leuk. 2018 Nov;18(11): $710-23$.

2 Steegmann JL, Baccarani $M$, Breccia $M$, Casado LF, García-Gutiérrez V, Hochhaus A, et al. European LeukemiaNet recommendations for the management and avoidance of adverse events of treatment in chronic myeloid leukaemia. Leukemia. 2016 Aug;30(8): 1648-71.

3 Rea D. Management of adverse events associated with tyrosine kinase inhibitors in chronic myeloid leukemia. Ann Hematol. 2015 Apr; 94(S2 Suppl 2):S149-58.

4 Palani R, Milojkovic D, Apperley JF. Managing pregnancy in chronic myeloid leukaemia. Ann Hematol. 2015 Apr;94(S2 Suppl 2):S16776.

5 Jiang Q, Liu ZC, Zhang SX, Gale RP. Young age and high cost are associated with future preference for stopping tyrosine kinase inhibitor therapy in Chinese with chronic myeloid leukemia. J Cancer Res Clin Oncol. 2016 Jul; 142(7):1539-47.

6 Marin D, Bazeos A, Mahon FX, Eliasson L, Milojkovic D, Bua M, et al. Adherence is the critical factor for achieving molecular responses in patients with chronic myeloid leukemia who achieve complete cytogenetic responses on imatinib. J Clin Oncol. 2010 May; 28(14):2381-8.
7 Noens L, van Lierde MA, De Bock R, Verhoef G, Zachée P, Berneman Z, et al. Prevalence, determinants, and outcomes of nonadherence to imatinib therapy in patients with chronic myeloid leukemia: the ADAGIO study. Blood. 2009 May;113(22):5401-11.

8 Cortes J, O'Brien S, Kantarjian H. Discontinuation of imatinib therapy after achieving a molecular response. Blood. 2004 Oct;104(7): 2204-5.

9 Mauro MJ, Druker BJ, Maziarz RT. Divergent clinical outcome in two CML patients who discontinued imatinib therapy after achieving a molecular remission. Leuk Res. 2004 May; 28 Suppl 1:S71-3.

10 Merante S, Orlandi E, Bernasconi P, Calatroni $S$, Boni M, Lazzarino M. Outcome of four patients with chronic myeloid leukemia after imatinib mesylate discontinuation. Haematologica. 2005 Jul;90(7):979-81.

11 Mahon FX, Réa D, Guilhot J, Guilhot F, Huguet F, Nicolini F, et al.; Intergroupe Français des Leucémies Myéloïdes Chroniques. Discontinuation of imatinib in patients with chronic myeloid leukaemia who have maintained complete molecular remission for at least 2 years: the prospective, multicentre Stop Imatinib (STIM) trial. Lancet Oncol. 2010 Nov;11(11):1029-35.

12 Mahon FX, Réa D, Guilhot J, Guilhot F, Huguet F, Nicolini FE, et al. Discontinuation of Imatinib in Patients with Chronic Myeloid Leukemia Who Have Maintained Complete
Molecular Response: Update Results of the STIM Study. American Society of Hematology 53rd Annual Meeting; 2011 Dec 10-13; San Diego, CA, USA. Blood. 2011;118(21):603.

13 Mahon FX, Réa D, Guilhot J, Guilhot F, Huguet F, Nicolini FE, et al. Long-Term Follow-Up after Imatinib Cessation for Patients in Deep Molecular Response: The Update Results Of The STIM1 Study. American Society of Hematology 55th Annual Meeting; 2013 Dec 7-13; New Orleans, LA, USA. Blood. 2013;122(21):255.

14 Ross M, Branford S, Seymour J, Arthur C, Schwarer A, Dang P, et al. Frequent and sustained drug-free remission in the Australian CML8 trial of imatinib withdrawal. 17th Congress of the European Hematology Association; 2012 Jun 14-17; Amsterdam, The Netherlands [abstract 0189]. Haematologica. 2012; 97 Suppl 1:74.

15 Ross DM, Branford S, Seymour JF, Schwarer AP, Arthur C, Yeung DT, et al. Safety and efficacy of imatinib cessation for CML patients with stable undetectable minimal residual disease: results from the TWISTER study. Blood. 2013 Jul;122(4):515-22.

16 Rousselot P, Charbonnier A, Cony-Makhoul P, Agape P, Nicolini FE, Varet B, et al. Loss of major molecular response as a trigger for restarting tyrosine kinase inhibitor therapy in patients with chronic-phase chronic myelogenous leukemia who have stopped imatinib after durable undetectable disease. J Clin Oncol. 2014 Feb;32(5):424-30. 
17 Hochhaus A, Masszi T, Giles FJ, Radich JP, Ross DM, Gómez Casares MT, et al. Treatment-free remission following frontline nilotinib in patients with chronic myeloid leukemia in chronic phase: results from the ENESTfreedom study. Leukemia. 2017 Jul;31(7): 1525-31.

18 Mahon FX, Boquimpani C, Kim DW, Benyamini N, Clementino NC, Shuvaev V, et al.; Treatment-Free Remission After SecondLine Nilotinib Treatment in Patients With Chronic Myeloid Leukemia in Chronic Phase Results From a Single-Group. TreatmentFree Remission After Second-Line Nilotinib Treatment in Patients With Chronic Myeloid Leukemia in Chronic Phase: Results From a Single-Group, Phase 2, Open-Label Study. Ann Intern Med. 2018 Apr;168(7):461-70.

19 Rea D, Nicolini FE, Tulliez M, Rousselot P, Guilhot F, Gardembas M, et al. Dasatinib or nilotinib discontinuation in chronic phase (CP)-chronic myeloid leukemia (CML) patients (pts) with durably undetectable BCRABL transcripts: interim analysis of the STOP 2G-TKI study with a minimum follow-up of 12 months - on behalf of the French CML Group Filmc. Blood. 2014;124(21). Abstract no. 811 .
20 Imagawa J, Tanaka $\mathrm{H}$, Okada $\mathrm{M}$, Nakamae $\mathrm{H}$, Hino M, Murai K, et al.; DADI Trial Group. Discontinuation of dasatinib in patients with chronic myeloid leukaemia who have maintained deep molecular response for longer than 1 year (DADI trial): a multicentre phase 2 trial. Lancet Haematol. 2015 Dec;2(12): e528-35.

21 Shah NP, García-Gutiérrez JV, JiménezVelasco A, Larson S, Saussele S, Rea D, et al. Updated 18-Month Results from Dasfree: A Study Evaluating Dasatinib Discontinuation in Patients (Pts) with Chronic Myeloid Leukemia in Chronic Phase (CML-CP) and Deep Molecular Response (DMR). Blood. 2018; 132:4253.

22 Breccia M, Foà R. Current Information and Recommendations on the Discontinuation of TKI Inhibitors in Chronic Myeloid Leukemia. Curr Oncol Rep. 2018 Mar;20(3):23.

23 Baccarani M, Deininger MW, Rosti G, Hochhaus A, Soverini S, Apperley JF, et al. European LeukemiaNet recommendations for the management of chronic myeloid leukemia: 2013. Blood. 2013 Aug;122(6):872-84.

24 Hughes TP, Ross DM. Moving treatment-free remission into mainstream clinical practice in CML. Blood. 2016 Jul;128(1):17-23.
25 Pallera A, Altman JK, Berman E, Abboud CN, Bhatnagar B, Curtin P, et al. NCCN Guidelines Insights: Chronic Myeloid Leukemia, Version 1.2017. J Natl Compr Canc Netw. 2016 Dec;14(12):1505-12.

26 Vardiman JW, Thiele J, Arber DA, Brunning RD, Borowitz MJ, Porwit A, et al. The 2008 revision of the World Health Organization (WHO) classification of myeloid neoplasms and acute leukemia: rationale and important changes. Blood. 2009 Jul;114(5):937-51.

27 Benghiat F, Beguin Y, Dessars B, Devos T, Lewalle $\mathrm{P}$, Mineur P, et al. Practical management of chronic myeloid leukaemia in Belgium. Belgian J Hematol. 2015;6(1):16-32.

28 Vrijens B, De Geest S, Hughes DA, Przemyslaw K, Demonceau J, Ruppar T, et al; ABC Project Team. A new taxonomy for describing and defining adherence to medications. Br J Clin Pharmacol. 2012 May;73(5):691-705.

29 Shash E, Bassi S, Cocorocchio E, Colpi GM, Cinieri S, Peccatori FA. Fatherhood during imatinib. Acta Oncol. 2011 Jun;50(5):734-5.

30 Oweini H, Otrock ZK, Mahfouz RA, Bazarbachi A. Successful pregnancy involving a man with chronic myeloid leukemia on dasatinib. Arch GynecolObstet.2011 Jan;283(1): 133-4. 